Collection SFN 10 (2010) 337-354

(C) Owned by the authors, published by EDP Sciences, 2010

DOI: $10.1051 / \mathrm{sfn} / 2010004$

\title{
Contribution magnétique à la fonction de diffusion des neutrons
}

\author{
F. Moussa et S. Petit \\ Institut Rayonnement Matière de Saclay Laboratoire Léon Brillouin, CE-Saclay, \\ 91190 Gif-sur-Yvette, France
}

\begin{abstract}
Résumé. Nous exposons les lois qui régissent les interactions entre les neutrons et les atomes dans le cadre de leur application à l'étude de la matière condensée. Nous nous intéressons plus particulièrement aux interactions magnétiques entre les neutrons et les électrons non appariés des atomes afin d'établir la section efficace de diffusion magnétique des neutrons.
\end{abstract}

\section{INTRODUCTION}

Dans une expérience de diffusion, les neutrons interagissent avec les noyaux de l'échantillon cible mais aussi avec le champ magnétique créé par le spin des électrons non appariés.

Ce processus magnétique est caractérisé par une certaine section efficace de diffusion, qui donne le nombre de neutrons déviés dans un angle solide $\Omega$ avec une énergie finale $E^{\prime}$. Le but de ce cours est de montrer comment on établit la forme de cette section efficace. Ceci nous permettra de montrer que les expériences de diffusion inélastique des neutrons permettent de mesurer très facilement, au moins dans le principe, les courbes de dispersion $\omega(\mathbf{q})$ des excitations magnétiques collectives dans la matière condensée. A titre d'illustration, nous aborderons l'exemple de systèmes isolants puis de systèmes métalliques. Dans une dernière partie, nous donnerons un bref aperçu des expériences de neutrons polarisés.

\section{INTERACTION MAGNÉTIQUE NEUTRON-ÉLECTRON}

Le potentiel d'interaction entre le moment magnétique du neutron $\mu_{n}$ et le champ magnétique $\mathbf{B}_{\mathbf{e}}$ créé par un électron s'écrit tout simplement (Figure 1):

$$
E_{n e}=-\mu_{n} \cdot \mathbf{B}_{e} .
$$

Ce champ est la somme de deux termes, provenant du moment magnétique intrinsèque de l'électron $\mu_{e}$ et d'une contribution orbitale liée à son mouvement (de vitesse $\mathbf{v}_{e}$ ) :

$$
\mathbf{B}_{e}(\mathbf{R})=\frac{\mu_{o}}{4 \pi}\left(\operatorname{rot}\left(\frac{\mu_{e} \times \mathbf{R}}{R^{3}}\right)-e \mathbf{v}_{e} \times \frac{\mathbf{R}}{R^{3}}\right) .
$$

On rappelle le lien entre moment magnétique et spin :

$$
\begin{aligned}
& \mu_{e}=-g \mu_{B} \mathbf{s}_{e} \text { avec } \mu_{B}=\frac{e \hbar}{2 m_{e}}, g \sim 2 \\
& \mu_{n}=-\gamma \mu_{N} \sigma_{n} \text { avec } \mu_{N}=\frac{e \hbar}{2 m_{n}}, \gamma \sim 1.91
\end{aligned}
$$

On rappelle $m_{n} / m_{e}=1838$ et donc $\mu_{N} \ll \mu_{B}$.

This is an Open Access article distributed under the terms of the Creative Commons Attribution-Noncommercial License 3.0, which permits unrestricted use, distribution, and reproduction in any noncommercial medium, provided the original work is properly cited. 


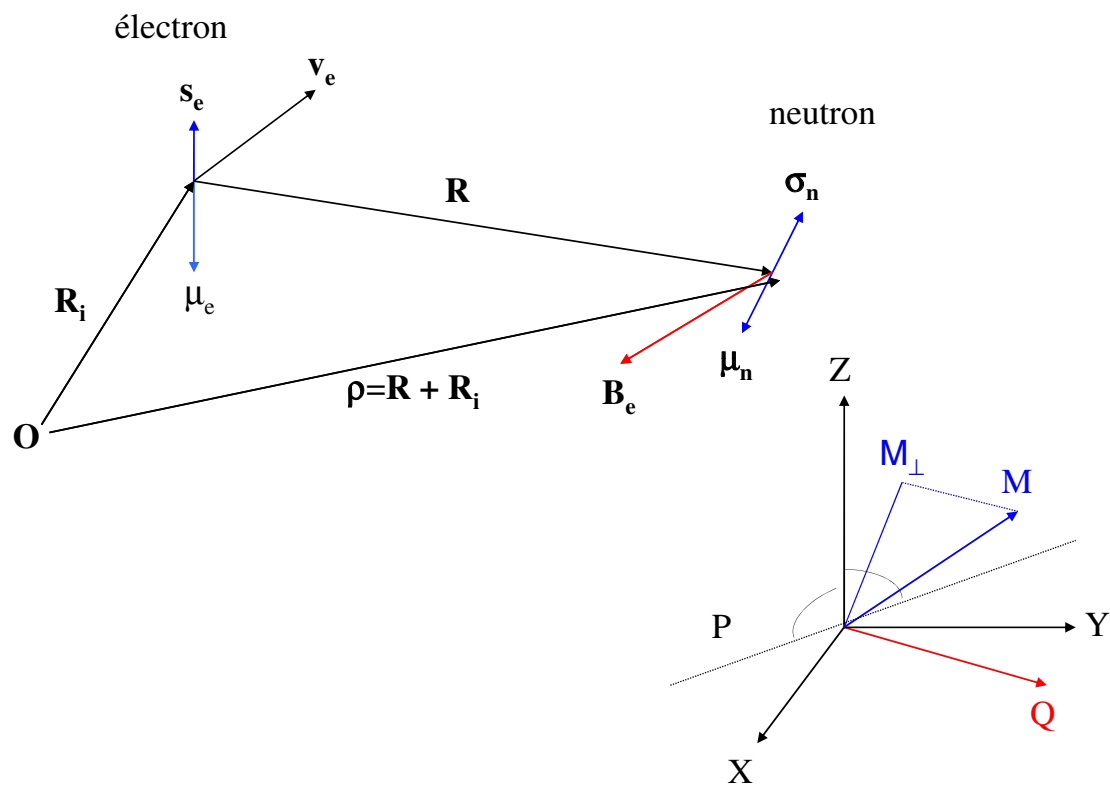

Figure 1. Champ magnétique $B_{e}$ et composantes de $M$ perpendiculaire à $Q$.

\section{SECTION EFFICACE DE DIFFUSION DES NEUTRONS}

Compte tenu de ce potentiel d'interaction, on détermine formellement la section efficace de diffusion des neutrons à l'aide de la règle d'or de Fermi :

$$
\frac{\partial^{2} \sigma}{\partial \Omega \partial E^{\prime}}=\frac{k^{\prime}}{k}\left(\frac{m_{n}}{2 \pi \hbar^{2}}\right)^{2} \sum_{\ell, \ell^{\prime}, \sigma, \sigma^{\prime}} p_{\ell} p_{\sigma}\left|\left\langle k^{\prime}, \ell^{\prime}, \sigma^{\prime}\left|E_{n e}\right| k, \ell, \sigma\right\rangle\right|^{2} \delta\left(\omega+E_{\ell}-E_{\ell^{\prime}}\right)
$$

avec les notations usuelles suivantes :

$\begin{array}{lll} & \text { Electron } & \text { Neutron } \\ \text { Etat initial } & |\ell\rangle, E_{\ell} & |k, \sigma\rangle, E=\frac{\hbar^{2} k^{2}}{2 m_{n}} \\ \text { Etat final } & \left|\ell^{\prime}\right\rangle, E_{\ell^{\prime}} & \left|k^{\prime}, \sigma^{\prime}\right\rangle, E^{\prime}=\frac{\hbar^{2} k^{2}}{2 m_{n}} .\end{array}$

La loi de conservation de l'énergie au cours du processus de diffusion impose :

$$
\omega=E_{\ell^{\prime}}-E_{\ell}=E-E^{\prime}
$$

On définit en outre le moment de transfert $\mathbf{Q}$ et le vecteur unitaire associé $\tilde{\mathbf{Q}}=\mathbf{Q} /|\mathbf{Q}|$. La suite du calcul de la section efficace est relativement fastidieux. En posant :

$$
r_{o}=4 \pi \frac{m_{n}}{2 \pi \hbar^{2}}\left(2 \mu_{N} \mu_{B} \frac{\mu_{o}}{4 \pi}\right)=\frac{\mu_{o} e^{2}}{4 \pi m_{e}}=0.5410^{-12} \mathrm{~cm}
$$


appelé rayon classique de l'électron, on obtient le résultat suivant :

$$
\begin{aligned}
\frac{\partial^{2} \sigma}{\partial \Omega \partial E^{\prime}} & =\frac{k^{\prime}}{k}\left(\gamma r_{o}\right)^{2} \sum_{\ell, \ell^{\prime}, \sigma, \sigma^{\prime}} p_{\ell} p_{\sigma}\left|\left\langle\ell^{\prime}, \sigma^{\prime}\left|\mathbf{r} \cdot \mathbf{M}_{\perp}\right| \ell, \sigma\right\rangle\right|^{2} \delta\left(\omega+E_{\ell}-E_{\ell^{\prime}}\right), \\
\mathbf{M}_{\perp} & =\sum_{i} e^{i \mathbf{Q} \mathbf{R}_{i}}\left(\tilde{\mathbf{Q}} \times\left(\mathbf{s}_{i} \times \tilde{\mathbf{Q}}\right)-\frac{i}{\hbar|\mathbf{Q}|} \tilde{\mathbf{Q}} \times \mathbf{p}_{i}\right) .
\end{aligned}
$$

$\mathbf{M}_{\perp}$ est un opérateur qui sélectionne la composante perpendiculaire à $\mathbf{Q}$ d'un opérateur aimantation construit à partir du spin $\mathbf{s}_{i}$ et de la quantité de mouvement $\mathbf{p}_{i}$ des électrons. C'est un premier résultat fondamental. On poursuit notre analyse en remarquant que par définition :

$$
\delta\left(\omega+E_{\ell}-E_{\ell^{\prime}}\right)=\frac{1}{2 \pi \hbar} \int d t e^{-i\left(\omega+E_{\ell}-E_{\ell^{\prime}}\right) t} .
$$

En appelant $H$ le Hamiltonien électronique, on a pour tout opérateur $O$ :

$$
O(t)=e^{i H t} O e^{-i H t} .
$$

On en déduit :

$$
\begin{aligned}
\left|\left\langle\ell^{\prime}|O| \ell\right\rangle\right|^{2} \delta\left(\omega+E_{\ell}-E_{\ell^{\prime}}\right) & =\frac{1}{2 \pi \hbar} \int d t\left\langle\ell|O| \ell^{\prime}\right\rangle\left\langle\ell^{\prime}|O| \ell\right\rangle e^{-i\left(\omega+E_{\ell}-E_{\ell^{\prime}}\right) t} \\
& =\frac{1}{2 \pi \hbar} \int d t\left\langle\ell|O| \ell^{\prime}\right\rangle\left\langle\ell^{\prime}|O(t)| \ell\right\rangle e^{-i \omega t} .
\end{aligned}
$$

Comme $\sum_{\ell^{\prime}}\left|\ell^{\prime}\right\rangle\left\langle\ell^{\prime}\right|=1$, on obtient la formule finale :

$$
\begin{aligned}
\frac{\partial^{2} \sigma}{\partial \Omega \partial E^{\prime}} & =\frac{k^{\prime}}{k}\left(\gamma r_{o}\right)^{2} \sum_{\ell, \sigma, \sigma^{\prime}} p_{\ell} p_{\sigma} \frac{1}{2 \pi \hbar} \int d t\left\langle\ell \sigma\left|\sigma \cdot \mathbf{M}_{\perp}(t=0)\right| \sigma^{\prime}\right\rangle\left\langle\sigma^{\prime}\left|\sigma \cdot \mathbf{M}_{\perp}(t)\right| \ell \sigma\right\rangle e^{-i \omega t}, \\
\mathbf{M}_{\perp}(t) & =\sum_{i} e^{i \mathbf{Q} \mathbf{R}_{i}(t)}\left(\tilde{\mathbf{Q}} \times\left(\mathbf{s}_{i}(t) \times \tilde{\mathbf{Q}}\right)-\frac{i}{\hbar|\mathbf{Q}|} \tilde{\mathbf{Q}} \times \mathbf{p}_{i}(t)\right) .
\end{aligned}
$$

Cette formule est bien difficile à utiliser dans des cas concrets. Nous allons donc voir dans les paragraphes suivants les simplifications que l'on utilise en pratique.

\subsection{Cas des neutrons non polarisés}

On envisage tout d'abord le cas fréquent où les neutrons incidents ne sont pas préparés dans un état de spin particulier. Nous verrons le cas complexe des neutrons polarisés à la fin de ce cours. On rappelle les définitions des matrices de Pauli :

$$
\sigma_{x}=\left(\begin{array}{ll}
0 & 1 \\
1 & 0
\end{array}\right) \quad \sigma_{y}=\left(\begin{array}{cc}
0 & -i \\
i & 0
\end{array}\right) \quad \sigma_{z}=\left(\begin{array}{cc}
1 & 0 \\
0 & -1
\end{array}\right)
$$

avec les propriétés :

$$
\begin{aligned}
\text { Trace } \sigma_{a} & =0, \\
\operatorname{det} \sigma_{a} & =-1,
\end{aligned}
$$




$$
\begin{aligned}
\sigma_{x} \sigma_{y} & =\sigma_{z}, \\
\sigma_{a} \sigma_{b}+\sigma_{b} \sigma_{a} & =2 \delta_{a b} .
\end{aligned}
$$

Dans ces conditions, on montre que :

$$
\sum_{\sigma, \sigma^{\prime}} p_{\sigma}\left\langle\sigma\left|\sigma_{a}\right| \sigma^{\prime}\right\rangle\left\langle\sigma^{\prime}\left|\sigma_{b}\right| \sigma\right\rangle=\delta_{a b}
$$

et par conséquent :

$$
\frac{\partial^{2} \sigma}{\partial \Omega \partial E^{\prime}}=\frac{k^{\prime}}{k}\left(\gamma r_{o}\right)^{2} \frac{1}{2 \pi \hbar} \int d t\left\langle\mathbf{M}_{\perp}(t=0) \cdot \mathbf{M}_{\perp}(t)\right\rangle e^{-i \omega t}
$$

\subsection{Cas où le magnétisme est dû aux spins des électrons}

Dans la plupart des cas, la contribution orbitale est négligeable. Dans ce cas on peut définir l'opérateur aimantation $\mathbf{M}_{\perp}(\mathbf{Q})$ uniquement à partir des spins des électrons non appariés :

$$
\begin{aligned}
\mathbf{M}_{\perp}(\mathbf{Q}) & =\sum_{i} e^{i \mathbf{Q} \mathbf{R}_{i}(t)}\left(\tilde{\mathbf{Q}} \times\left(\mathbf{s}_{i}(t) \times \tilde{\mathbf{Q}}\right)\right) \\
& =\sum_{\alpha, \beta}\left(\delta_{\alpha, \beta}-\tilde{Q}_{\alpha} \tilde{Q}_{\beta}\right) \sum_{i} e^{i \mathbf{Q} \mathbf{R}_{i}(t)} s_{i}^{\beta}(t)
\end{aligned}
$$

La position $\mathbf{R}_{i}$ d'un tel électron peut se décomposer comme suit :

$$
\mathbf{R}_{i}=\mathbf{R}_{\ell, d}+\mathbf{r}_{v}
$$

où $\mathbf{R}_{\ell, d}$ identifie l'ion $d$ dans la maille $\ell$ et $\mathbf{r}_{v}$ celle de l'électron dans l'ion. On a donc :

$$
\sum_{\ell, d} e^{i \mathbf{Q} \mathbf{R}_{\ell, d}} \sum_{\nu} e^{i \mathbf{Q} \mathbf{r}_{v}} \mathbf{s}_{v}=\sum_{\ell, d} e^{i \mathbf{Q R}_{\ell, d}} \int d^{3} r e^{i \mathbf{Q r}}\left(\sum_{\nu} \delta\left(\mathbf{r}-\mathbf{r}_{v}\right) \mathbf{s}_{v}\right) .
$$

La quantité $\sum_{v} \delta\left(\mathbf{r}-\mathbf{r}_{v}\right) \mathbf{s}_{v}$ est la densité de spin des électrons de l'ion. Mais du fait de la règle de Hund, le spin résultant est la somme des spins des électrons non appariés. On va donc pouvoir définir un spin effectif sur le site $\ell, d$ noté $\mathbf{S}_{\ell, d}$ ainsi qu'une densité $\rho$ de telle sorte que :

$$
\mathbf{S}_{\ell, d} \int d^{3} r e^{i \mathbf{Q r}} \rho(\mathbf{r})=\int d^{3} r e^{i \mathbf{Q r}} \sum_{\nu} \delta\left(\mathbf{r}-\mathbf{r}_{\nu}\right) \mathbf{S}_{\nu} .
$$

La quantité

$$
F(\mathbf{Q})=\int d^{3} r e^{i \mathbf{Q} \mathbf{r}} \rho(\mathbf{r})
$$

est appelé facteur de forme de l'ion $d$. Il est tabulé en fonction de $|\mathbf{Q}|$ dans les tables internationales. On remarque que $F(0)=1$. La section efficace s'écrit donc :

$$
\begin{aligned}
\frac{\partial^{2} \sigma}{\partial \Omega \partial E^{\prime}}= & \frac{k^{\prime}}{k}\left(\gamma r_{o}\right)^{2} \frac{1}{2 \pi \hbar} \sum_{\ell, \ell^{\prime}, d, d^{\prime}} F_{d}(\mathbf{Q}) F_{d^{\prime}}^{*}(\mathbf{Q}) \\
& \times \int d t\left\langle e^{i \mathbf{Q} \mathbf{R}_{\ell, d}} e^{-i \mathbf{Q} \mathbf{R}_{\ell^{\prime}, d^{\prime}}(t)} \mathbf{S}_{\perp, \ell, d}(t=0) . \mathbf{S}_{\perp, \ell^{\prime}, d^{\prime}}(t)\right\rangle e^{-i \omega t} .
\end{aligned}
$$




\subsection{Cas où le magnétisme est dû au spin et au moment orbital des électrons}

Si le moment orbital $\mathbf{L}$ n'est pas bloqué, il faut en tenir compte dans la contribution magnétique à la section efficace de diffusion. Le calcul est complexe et nous présentons ici une forme simplifiée (pour les terres rares par exemple, voir S.W. Lovesey vol. 2 chap. 11). On fait l'approximation dipolaire valable si la fonction d'onde des électrons ne s'étend pas au-delà de $1 /|\mathbf{Q}|$. La quantité importante est maintenant le moment cinétique total $\mathbf{J}=\mathbf{L}+\mathbf{S}$. A l'intérieur de l'espace formé par les $2 J+1$ vecteurs décrivant l'état du moment cinétique $\mathbf{J}$, les éléments de matrice de tout opérateur vectoriel sont proportionnels aux éléments de matrice de $\mathbf{J}$, en vertu du théorème de Wigner-Eckart. La contribution magnétique d'un spin $\mathbf{S}$ est $2 \mu_{B} \mathbf{S}$ et celle d'un moment orbital $\mathbf{L}$ est $\mu_{B} \mathbf{L}$. On construit donc l'opérateur $g \mathbf{J}=\mathbf{L}+2 \mathbf{S}$. La section efficace ci-dessus reste valable à condition de remplacer $\mathbf{S}$ par $g \mathbf{J} / 2$. Le facteur de forme $F(\mathbf{Q})$ est formellement identique mais il est maintenant associé à la densité de $g \mathbf{J} / 2$.

$$
\begin{aligned}
\frac{\partial^{2} \sigma}{\partial \Omega \partial E^{\prime}}= & \frac{k^{\prime}}{k}\left(\gamma r_{o}\right)^{2} \frac{1}{2 \pi \hbar} \sum_{\ell, \ell^{\prime}, d, d^{\prime}} \frac{g F_{d}(\mathbf{Q})}{2} \frac{g F_{d^{\prime}}^{*}(\mathbf{Q})}{2} \\
& \times \int d t\left\langle e^{i \mathbf{Q} \mathbf{R}_{\ell, d}} e^{-i \mathbf{Q} \mathbf{R}_{\ell^{\prime}, d^{\prime}}(t)} \mathbf{J}_{\perp, \ell, d}(t=0) \cdot \mathbf{J}_{\perp, \ell^{\prime}, d^{\prime}}(t)\right\rangle e^{-i \omega t} .
\end{aligned}
$$

\subsection{Couplage entre corrélations de spin et corrélations de position}

Nous avons vu plus haut que la section efficace de diffusion des neutrons fait intervenir l'opérateur $\mathbf{M}_{\perp}(t)$ et par conséquent les opérateurs spin ET les opérateurs position $\mathbf{R}_{i}$ pris au temps $t$. Ce mélange entre opérateur de spin et opérateur de position est la source de sérieuses complications. Pour s'en sortir, on est amené à faire de nouvelles hypothèses énoncées ci-dessous :

- les ions sont supposés effectuer des oscillations harmoniques de faible amplitude $\left(\mathbf{u}_{\ell, d}\right)$ autour de leur position d'équilibre $\mathbf{R}_{\ell, d}^{o}$ :

$$
\mathbf{R}_{\ell, d}(t)=\mathbf{R}_{\ell, d}^{o}+\mathbf{u}_{\ell, d}
$$

Nous aurons donc à calculer :

$$
\sum_{\ell, d, \ell^{\prime}, d^{\prime}} F_{d}(\mathbf{Q}) F_{d^{\prime}}^{*}(\mathbf{Q}) e^{i \mathbf{Q}\left(\mathbf{R}_{\ell, d}^{o}-\mathbf{R}_{\ell^{\prime}, d^{\prime}}^{o}\right)} \int d t\left\langle e^{i \mathbf{Q} \mathbf{u}_{\ell, d}} e^{-i \mathbf{Q} \mathbf{u}_{\ell^{\prime}, d^{\prime}}(t)} \mathbf{S}_{\perp, \ell, d}(t=0) \cdot \mathbf{S}_{\perp, \ell^{\prime}, d^{\prime}}(t)\right\rangle e^{-i \omega t}
$$

- on suppose que le mouvement des ions et l'orientation des spins sont décorrélés; on a donc :

$$
\left\langle e^{i \mathbf{Q} \mathbf{u}_{\ell, d}} e^{-i \mathbf{Q} \mathbf{u}_{\ell^{\prime}, d^{\prime}}(t)} \mathbf{S}_{\perp, \ell, d}(t=0) \cdot \mathbf{S}_{\perp, \ell^{\prime}, d^{\prime}}(t)\right\rangle=\left\langle e^{i \mathbf{Q} \mathbf{u}_{\ell, d}} e^{-i \mathbf{Q} \mathbf{u}_{\ell^{\prime}, d^{\prime}}(t)}\right\rangle\left\langle\mathbf{S}_{\perp, \ell, d}(t=0) \cdot \mathbf{S}_{\perp, \ell^{\prime}, d^{\prime}}(t)\right\rangle
$$

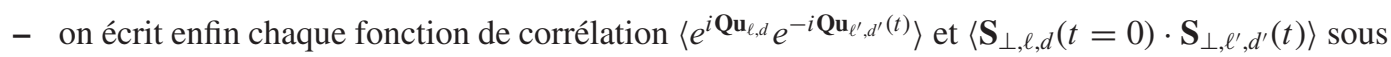
la forme d'une somme de 2 termes, le premier correspondant à sa valeur asymptotique au temps infini, l'autre à l'écart entre la valeur au temps $t$ et cette valeur asymtpotique.

L'expression que l'on doit retenir pour la section efficace de diffusion magnétique correspond à la formule obtenue dans l'hypothèse où la fonction de corrélation relative aux positions atomiques est remplacée par sa valeur asymptotique :

$$
\left\langle e^{i \mathbf{Q} \mathbf{u}_{\ell, d}} e^{-i \mathbf{Q} \mathbf{u}_{\ell^{\prime}, d^{\prime}}(t)}\right\rangle \approx\left\langle e^{i \mathbf{Q} \mathbf{u}_{\ell, d}}\right\rangle\left\langle e^{-i \mathbf{Q} \mathbf{u}_{\ell^{\prime}, d^{\prime}}}\right\rangle .
$$

Dans la mesure où les ions ont un mouvement harmonique, on reconnait l'expression du facteur de Debye Waller $W_{\ell, d}$ (voir le cours de H. Schober). La section efficace de diffusion magnétique 
devient donc :

$$
\begin{aligned}
\frac{\partial^{2} \sigma}{\partial \Omega \partial E^{\prime}}= & \frac{k^{\prime}}{k}\left(\gamma r_{o}\right)^{2} \frac{1}{2 \pi \hbar} \sum_{\ell, \ell^{\prime}, d, d^{\prime}} e^{i \mathbf{Q}\left(\mathbf{R}_{\ell, d}^{o}-\mathbf{R}_{\ell^{\prime}, d^{\prime}}^{o}\right)} F_{d}(\mathbf{Q}) e^{-W_{\ell, d}} F_{d}^{*}(\mathbf{Q}) e^{-W_{\ell^{\prime}, d^{\prime}}} \\
& \times \int d t\left\langle\mathbf{S}_{\perp, \ell, d}(t=0) \cdot \mathbf{S}_{\perp, \ell^{\prime}, d^{\prime}}(t)\right\rangle e^{-i \omega t} .
\end{aligned}
$$

L'expression ainsi obtenue tient compte du "désordre" de position lié à l'agitation thermique, ce que traduit le facteur de Debye. Rappelons qu'il est parfois utile d'écrire explicitement $\mathbf{S}_{\perp}$ en fonction de $\mathbf{S}$ :

$$
S_{\perp}^{a}=\sum_{b}\left(\delta_{a b}-\tilde{Q}^{a} \tilde{Q}^{b}\right) S^{b}
$$

et

$$
\begin{aligned}
\mathbf{S}_{\perp} \cdot \mathbf{S}_{\perp} & =\sum_{a} S_{\perp}^{a} S_{\perp}^{a} \\
& =\sum_{a} S^{a} S^{a}-\sum_{a, b} 2 \tilde{Q}^{a} \tilde{Q}^{b} S^{a} S^{b}+\sum_{a, b, c} \tilde{Q}^{a} \tilde{Q}^{b} \tilde{Q}^{a} \tilde{Q}^{c} S^{b} S^{c} \\
& =\sum_{a} S^{a} S^{a}-\sum_{a, b} 2 \tilde{Q}^{a} \tilde{Q}^{b} S^{a} S^{b}+\sum_{b, c} \tilde{Q}^{b} \tilde{Q}^{c} S^{b} S^{c} \\
& =\sum_{a} S^{a}\left(\delta_{a b}-\tilde{Q}^{a} \tilde{Q}^{b}\right) S^{b} .
\end{aligned}
$$

\subsubsection{Remarque}

La "vraie" section efficace contient bien entendu d'autres termes, et en particulier, le pendant de celui que nous venons de décrire et que l'on obtient en remplaçant la fonction de corrélation de spin par sa valeur asymtpotique :

$$
\left\langle\mathbf{S}_{\perp, \ell, d}(t=0) \cdot \mathbf{S}_{\perp, \ell^{\prime}, d^{\prime}}(t)\right\rangle \approx\left\langle\mathbf{S}_{\perp, \ell, d}(t=0) \cdot \mathbf{S}_{\perp, \ell^{\prime}, d^{\prime}}(t=\infty)\right\rangle=\left\langle\mathbf{S}_{\perp, \ell, d}\right\rangle \cdot\left\langle\mathbf{S}_{\perp, \ell^{\prime}, d^{\prime}}\right\rangle
$$

ce qui donne une nouvelle contribution :

$$
\begin{aligned}
\frac{\partial^{2} \sigma}{\partial \Omega \partial E^{\prime}}= & \frac{k^{\prime}}{k}\left(\gamma r_{o}\right)^{2} \frac{1}{2 \pi \hbar} \sum_{\ell, \ell^{\prime}, d, d^{\prime}} F_{d}(\mathbf{Q}) F_{d^{\prime}}^{*}(\mathbf{Q})\left\langle\mathbf{S}_{\perp, \ell, d}\right\rangle\left\langle\mathbf{S}_{\perp, \ell^{\prime}, d^{\prime}}\right\rangle e^{i \mathbf{Q}\left(\mathbf{R}_{\ell, d}^{o}-\mathbf{R}_{\ell^{\prime}, d^{\prime}}^{o}\right)} \\
& \times \int d t\left\langle e^{i \mathbf{Q} \mathbf{u}_{\ell, d}} e^{-i \mathbf{Q} \mathbf{u}_{\ell^{\prime}, d^{\prime}}(t)}\right\rangle e^{-i \omega t}
\end{aligned}
$$

Celle-ci ressemble formellement à ce que nous avons appelé section efficace de diffusion des neutrons par les ions seuls dans le cours de H. Schober et définie par :

$$
\frac{\partial^{2} \sigma}{\partial \Omega \partial E^{\prime}}=\frac{k^{\prime}}{k} \frac{1}{2 \pi \hbar} \sum_{\ell, \ell^{\prime}, d, d^{\prime}} b_{d} b_{d^{\prime}} e^{i \mathbf{Q}\left(\mathbf{R}_{\ell, d}^{o}-\mathbf{R}_{\ell^{\prime}, d^{\prime}}^{o}\right)} \int d t\left\langle e^{i \mathbf{Q} \mathbf{u}_{\ell, d}} e^{-i \mathbf{Q u}_{\ell^{\prime}, d^{\prime}}(t)}\right\rangle e^{-i \omega t}
$$

La quantité

$$
\left(\gamma r_{o}\right)^{2} F_{d}(\mathbf{Q}) F_{d^{\prime}}^{*}(\mathbf{Q}) e^{i \mathbf{Q}\left(\mathbf{R}_{\ell, d}^{o}-\mathbf{R}_{\ell^{\prime}, d^{\prime}}^{o}\right)}\left\langle\mathbf{S}_{\perp, \ell, d}(t=0) \cdot \mathbf{S}_{\perp, \ell^{\prime}, d^{\prime}}(t \rightarrow \infty)\right\rangle
$$


apparait comme une sorte de renormalisation des longueurs de diffusion. Pour illustrer ce point, prenons l'exemple d'un composé ferromagnétique dont les spins sont alignés selon l'axe $z$. Nous avons donc :

$$
\left\langle\mathbf{S}_{\perp, \ell, d}(t=0) \cdot \mathbf{S}_{\perp, \ell^{\prime}, d^{\prime}}(t \rightarrow \infty)\right\rangle=S^{2}\left(1-\frac{\tilde{Q}_{z}^{2}}{\tilde{Q}^{2}}\right)
$$

et cette contribution à la section efficace de diffusion magnétique devient :

$$
\frac{\partial^{2} \sigma}{\partial \Omega \partial E^{\prime}}=\frac{k^{\prime}}{k} \frac{1}{2 \pi \hbar} \int d t \sum_{\ell, \ell^{\prime}, d, d^{\prime}}\left(\left(\gamma r_{o}\right)^{2} F_{d}(\mathbf{Q}) F_{d^{\prime}}^{*}(\mathbf{Q}) S^{2}\left(1-\frac{\tilde{Q}_{z}^{2}}{\tilde{Q}^{2}}\right)\right)\left\langle e^{i \mathbf{Q} \mathbf{R}_{\ell, d}} e^{-i \mathbf{Q R}_{\ell^{\prime}, d^{\prime}}(t)}\right\rangle e^{-i \omega t} .
$$

Tout se passe bien comme si on ajoutait à la longueur de diffusion de l'ion $\ell, d$ la contriution :

$$
b_{\text {eff }}=\gamma r_{o} F_{d}(\mathbf{Q}) S \sqrt{1-\frac{\tilde{Q}_{z}^{2}}{\tilde{Q}^{2}}}
$$

En particulier, les modes de phonons vont être habillés par cette contribution magnétique. On pourrait les confondre avec les ondes de spin, mais heureusement, le facteur géométrique $1-\frac{\tilde{Q}_{z}^{2}}{\tilde{Q}^{2}}$ empêche cette confusion : en effet, les ondes de spin sont des fluctuations des composantes de spin perpendiculaires au moment ordonné, soit l'axe $z$. Pour les mesurer, on doit donc prendre $Q / / z$, de sorte que dans ce cas $1-\frac{\tilde{Q}_{z}^{2}}{\tilde{Q}^{2}}=0$ et $b_{\text {eff }}=0$.

\section{SECTION EFFICACE DANS LE CAS D'UN FERROMAGNÉTIQUE ISOLANT}

Pour mettre en musique ces développements mathématiques, on se propose d'étudier le cas simple d'un isolant ferromagnétique, avec un seuil ion magnétique par maille, et que l'on suppose bien décrit par un modèle de Heisenberg :

$$
H=\sum_{m, n} J_{m, n} \mathbf{S}_{m} \mathbf{S}_{n}
$$

Ce système présente un ordre magnétique à grande distance (les spins s'orientent selon z) qui donne une contribution statique (des pics de Bragg) dans la section efficace de diffusion des neutrons. Le calcul montre que les composantes longitudinales du spin (selon $z$ ) donnent une contribution purement élastique :

$$
\begin{aligned}
\sum_{m, n} e^{i \mathbf{Q}\left(\mathbf{R}_{m}-\mathbf{R}_{n}\right)}\left\langle S_{m}^{z} S_{n}^{z}\right\rangle & =\sum_{m, n} e^{i \mathbf{Q}\left(\mathbf{R}_{m}-\mathbf{R}_{n}\right)} S^{2} \\
& =\frac{(2 \pi)^{3}}{v_{o}} S^{2} \sum_{\tau} \delta(\mathbf{Q}-\tau)
\end{aligned}
$$

où $\tau$ est un vecteur du réseau réciproque magnétique. On obtient donc :

$$
\frac{\partial^{2} \sigma}{\partial \Omega \partial E^{\prime}}=\left(\gamma r_{o}\right)^{2} S^{2}\left(1-\frac{\tilde{Q}_{z}^{2}}{\tilde{Q}^{2}}\right) \frac{(2 \pi)^{3}}{v_{o}} \sum_{\tau} \delta(\mathbf{Q}-\tau) \delta(\omega)
$$

Pour pouvoir observer ces pics de Bragg, c'est-à-dire observer les composantes de spin selon $z$, on vérifie qu'il faut choisir $\mathbf{Q}$ perpendiculaire à $z$. Ce système présente également des excitations collectives, que l'on appelle des "ondes de spin". Physiquement, un tel état excité se caractérise par une composante du spin selon $z$ légèrement réduite au profit d'une petite composante "transverse", c'est-à-dire contenue dans le plan $x y$, et qui oscille dans le temps. Ces mouvements de précession corrélés de site en site, forment une onde de vecteur d'onde q et de fréquence (ou d'énergie) $\omega$. Leur dispersion, la loi $\omega(\mathbf{q})$, 
contient naturellement des informations sur les constantes de couplage $J_{m, n}$. En se basant sur le calcul de la section efficace de diffusion inélastique des neutrons dans le cadre de ce modèle, nous allons voir qu'il est possible de mesurer cette dispersion.

\subsection{Les ondes de spin}

Suivant la méthode dite de Holstein-Primakov, on introduit des opérateurs bosons :

$$
\begin{aligned}
S_{m}^{+}=S_{m}^{x}+i S_{m}^{y} & =\sqrt{2 S-n_{b}} b_{m}, \\
S_{m}^{-}=S_{m}^{x}-i S_{m}^{2} & =b_{m}^{+} \sqrt{2 S-n_{b}}, \\
S_{m}^{z} & =S-n_{b} .
\end{aligned}
$$

de sorte que le spin s'écrit (au premier ordre) :

$$
\mathbf{S}_{m}=\left(\begin{array}{cc}
\frac{\sqrt{2 S}}{2} & \left(b_{m}+b_{m}^{+}\right) \\
\frac{\sqrt{2 S}}{2 i} & \left(b_{m}-b_{m}^{+}\right) \\
S-n_{b}
\end{array}\right)
$$

Après quelques calculs, au deuxième ordre dans ces opérateurs, on obtient l'Hamiltonien des ondes de spin :

$$
H=-S \sum_{m}\left(\sum_{n} J_{m, n}\right)\left(b_{m}^{+} b_{m}+b_{m} b_{m}^{+}\right)+S J_{m, n}\left(b_{m}^{+} b_{n}+b_{m} b_{n}^{+}\right) .
$$

On achève la diagonalisation de $H$ par une transformation de Fourier :

$$
H=\sum_{\mathbf{q}}-S J\left(\zeta-\sum_{\Delta} \cos \mathbf{q} \Delta\right) b_{q}^{+} b_{q}
$$

où $\zeta$ est le nombre de voisins et $\Delta$ un vecteur pointant vers un de ces proches voisins. La loi de dispersion des ondes des ondes de spin est donc

$$
\omega_{\mathbf{q}}=-S J\left(\zeta-\sum_{\Delta} \cos \mathbf{q} \Delta\right)
$$

\subsection{Section efficace de diffusion}
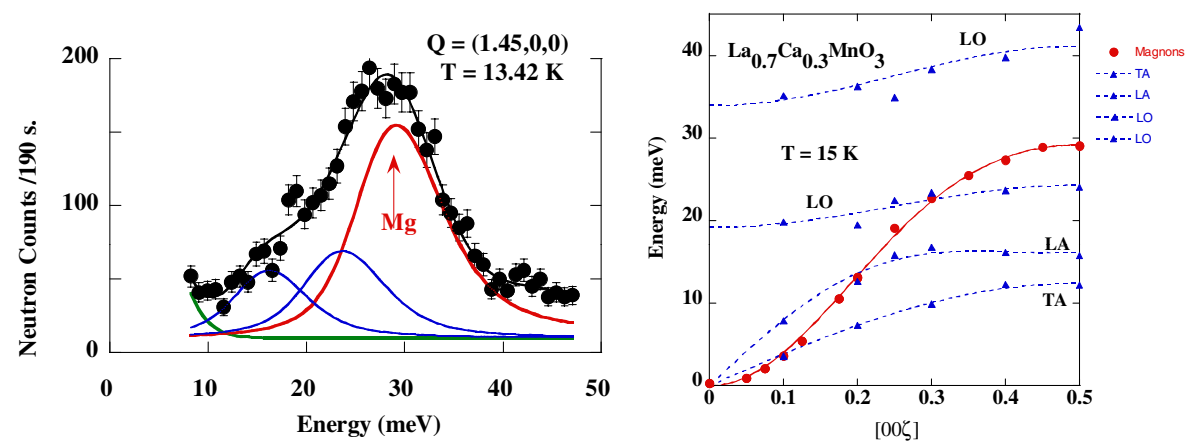

Figure 2. A gauche : spectre à Q constant en fonction de l'énergie. A droite : courbes de dispersion des ondes de spin (en rouge) et des phonons (en bleu) à $\mathrm{T}=13 \mathrm{~K}$. 
Le calcul des fonctions de corrélation spin-spin (selon x et y) s'effectue de la manière suivante :

$$
\begin{aligned}
\int d t e^{-i \omega t} \sum_{m, n} e^{i \mathbf{Q}\left(\mathbf{R}_{m}-\mathbf{R}_{n}\right)}\left\langle S_{m}^{x} S_{n}^{x}(t)\right\rangle & =\int d t e^{-i \omega t} \sum_{\mathbf{q}, \tau} \delta(\mathbf{Q}-\mathbf{q}-\tau) \frac{S}{2}\left\langle b_{\mathbf{q}} b_{\mathbf{q}}^{+} e^{i \omega_{\mathbf{q}} t}+b_{\mathbf{q}}^{+} b_{\mathbf{q}} e^{-i \omega_{\mathbf{q}} t}\right\rangle \\
& =\frac{S}{2} \sum_{\mathbf{q}, \tau} \delta(\mathbf{Q}-\mathbf{q}-\tau)\left(\left(1+n_{\mathbf{q}}\right) \delta\left(\omega-\omega_{\mathbf{q}}\right)+n_{\mathbf{q}} \delta\left(\omega+\omega_{\mathbf{q}}\right)\right)
\end{aligned}
$$

En effet, l'équation du mouvement de l'opérateur $b_{\mathbf{q}}^{+}$s'écrit :

$$
i \frac{d b_{\mathbf{q}}^{+}}{d t}=\left[b_{\mathbf{q}}^{+}, H\right]=-\omega_{\mathbf{q}} b_{\mathbf{q}}^{+}
$$

et donc :

$$
b_{\mathbf{q}}^{+}(t)=e^{i \omega t} b_{\mathbf{q}}^{+} .
$$

On dit que l'opérateur $b_{\mathbf{q}}^{+}$crée une excitation élémentaire, soit une onde de spin d'énergie $\omega_{\mathbf{q}}$. Dans ces expressions, on voit apparaitre le facteur de Bose pour le mode $\omega_{\mathbf{q}}$ :

$$
\omega_{\mathbf{q}}=\frac{1}{e^{\frac{\hbar \omega_{\mathbf{q}}}{k_{B} T}}-1} .
$$

Tenant compte des composantes perpendiculaires à $\mathbf{Q}$, on obtient finalement :

$$
\begin{aligned}
\frac{\partial^{2} \sigma}{\partial \Omega \partial E^{\prime}}= & \frac{k^{\prime}}{k}\left(\gamma r_{o}\right)^{2}\left|F(\mathbf{Q}) e^{-W(\mathbf{Q})}\right|^{2} \frac{S}{2}\left(1+\frac{\tilde{Q}_{z}^{2}}{\tilde{Q}^{2}}\right) \\
& \times \sum_{\mathbf{q}, \tau} \delta(\mathbf{Q}-\mathbf{q}-\tau)\left(\left(1+n_{\mathbf{q}}\right) \delta\left(\omega-\omega_{\mathbf{q}}\right)+n_{\mathbf{q}} \delta\left(\omega+\omega_{\mathbf{q}}\right)\right) .
\end{aligned}
$$

Cette expression nous permet de tirer les conclusions suivantes :

- En balayant l'espace des énergies dans différentes directions de symétrie du cristal, on peut construire pas à pas la courbe de dispersion $\omega_{\mathbf{q}}$. Ces mesures se font typiquement sur un spectromètre 3 axes.

- Le facteur géométrique $\left(1+\frac{\tilde{Q}_{z}^{2}}{\tilde{Q}^{2}}\right)$ illustre le fait que pour bien observer les ondes de spin, qui sont des fluctuations des composantes de spin contenues dans le plan $x y$, il faut faire les mesures avec $Q$ perpendiculaire au plan $x y$, et donc le plus possible selon $z$.

- Dans le cas d'un simple ferromagnétique, il n'y a pas de facteur de structure à proprement parler. Ceci n'est plus vrai dans le cas général (le formalisme est exposé dans l'article de S. Petit), mais la forme de la section efficace avec en particulier des fonctions $\delta\left(\omega \pm \omega_{\mathbf{q}}\right)$ reste vraie. Dans tous les cas, l'intensité varie avec le facteur de forme magnétique et diminue rapidement avec $|\mathbf{Q}|$. Les mesures se feront donc aux faibles valeurs de $|\mathbf{Q}|$.

- Quand le ferromagnétisme n'existe qu'à basse température, le facteur de Bose est faible pour des énergies $\omega_{\mathbf{q}} \ll k_{B} T$. Dans ce cas l'étude des ondes de spin se fait uniquement en création, pour profiter du 1 dans le facteur $1+n_{\mathbf{q}}$.

- Quand on s'approche de la température de Curie, les ondes de spin ne sont plus des ondes planes, mais plutôt des paquets d'onde en interaction. On peut les modéliser par des ondes planes amorties. Si l'amortissement n'est pas trop grand devant l'énergie du mode, $\Gamma \ll \omega_{\mathbf{q}}$, l'intensité inélastique est bien décrite par le modèle de l'oscillateur amorti ou encore par une lorentzienne. 
Dans ce cas on remplace dans la section efficace, la fonction $\delta\left(\omega-\omega_{\mathbf{q}}\right)$ par

$$
\frac{\omega^{2} \Gamma^{2}}{\left(\omega^{2}-\omega_{\mathbf{q}}^{2}\right)^{2}+(\omega \Gamma)^{2}}
$$

ou par

$$
\frac{\omega \Gamma}{\left(\omega^{2}-\omega_{\mathbf{q}}^{2}\right)+(\omega \Gamma)},
$$

ce qui est équivalent dans le cas d'un faible amortissement.

La figure 2 présente un exemple de spectre à $\mathbf{Q}$ constant $\mathbf{Q}=\tau+\mathbf{q}, \tau=(1,0,0), \mathbf{q}=(0.45,0,0)$ et énergie variable. Celui-ci fait apparaitre trois modes; le plus intense est le magnon à $29 \mathrm{meV}$, mais on distingue deux autres modes moins intenses que l'on identifie comme des phonons. Un test simple pour s'en assurer consiste à chauffer l'échantillon. Les magnons se renormalisent à $T_{c}$ alors que les phonons n'évoluent que très lentement avec la température. Si le doute persiste, on peut utiliser des neutrons polarisés. De tels spectres permettent de déterminer les courbes de dispersion des ondes de spin dans différentes directions de symétrie. Si on dispose d'un modèle auquel on peut les comparer, par exemple le modèle de Heisenberg, on pourra en déduire les intégrales d'échange entre voisins. Ici, la dispersion des ondes de spin (en rouge) est bien représentée par un couplage $J_{1}$ entre premiers voisins (distants de a) et $J_{4}$ entre quatrièmes voisins (distants de $2 \mathrm{a}$ ). On trouve $J_{1}=1.83 \mathrm{meV}$ et $J_{4}=0.23 \mathrm{meV}$ (En bleu les branches de phonons).

\section{LE CAS D'UN MÉTAL}

Les mesures de diffusion inélastique des neutrons permettent également de déterminer les excitations collectives dans les métaux. Le magnétisme n'est plus "localisé", c'est-à-dire induit par des électrons localisés; au contraire, nous avons affaire à un magnétisme de bandes électroniques. Les excitations collectives ne correspondent plus du tout à un mouvement de précession des spins; il faut plutôt les imaginer comme des modulations de la densité électronique, et donc de la densité de spin ( $\uparrow$ ou $\downarrow$ ).

\subsection{La susceptibilité généralisée}

Dans ce type de problèmes, on utilise souvent, à la place des fonctions de corrélation, la notion de susceptibilité généralisée $\chi(\mathbf{q}, \omega)$. Cette quantité lie l'aimantation $\mathbf{M}(\mathbf{q}, \omega)$ d'un système en réponse à une sollicitation externe (un champ magnétique) $\mathbf{H}(q, \omega)$ :

$$
M^{a}(\mathbf{q}, \omega)=\chi^{a b}(\mathbf{q}, \omega) H^{b}(\mathbf{q}, \omega) .
$$

Toutefois, en vertu du théorème de fluctuation-dissipation, ces deux manières d'aborder les problèmes sont équivalentes. Mathématiquement, elles sont liées par la relation :

$$
\left\langle S^{a} S^{b}\right\rangle=(1+n(\omega)) \chi_{a b}^{\prime \prime}(\mathbf{q}, \omega)
$$

de sorte que :

$$
\frac{\partial^{2} \sigma}{\partial \Omega \partial E^{\prime}}=\frac{k^{\prime}}{k}\left(\gamma r_{o}\right)^{2}\left|F(\mathbf{Q}) e^{-W(\mathbf{Q})}\right|^{2}(1+n(\omega)) \sum_{a, b}\left(\delta_{a b}-\tilde{Q}^{a} \tilde{Q}^{b}\right) \chi_{a b}^{\prime \prime}
$$

Le calcul de la susceptibilité repose le plus souvent sur l'approximation dite de la RPA ("random phase approximation") : pour tenir compte des corrélations entre électrons, on ajoute au champ externe, le champ interne $I(\mathbf{q}) \mathbf{M}(\mathbf{q}, \omega)$ induit par ces corrélations (voir le cours de S. Raymond). Si $\chi_{o}$ est la 
susceptibilité du système sans corrélations, on écrit donc la relation entre l'aimantation et le champ externe sous la forme :

$$
\mathbf{M}=\chi \mathbf{H}=\chi_{o}(\mathbf{H}+I \mathbf{M}) .
$$

On en déduit donc $\mathbf{M}=\left(1-\chi_{o} I\right)^{-1} \times \chi_{o} \times \mathbf{H}$ et la susceptibilité devient :

$$
\chi=\left(1-\chi_{o} I\right)^{-1} \chi_{o}
$$

Chaque fois que $1 \approx \chi_{o} I$, nous aurons donc un signal "intense" dans la section efficace de diffusion. L'ensemble de ces points peut dessiner une ligne $\omega(\mathbf{q})$ dans l'espace, et donc l'équivalent de la relation de dispersion obtenue dans le cas "isolant". A chacun de ces points, ou pôles, correspond une excitation collective du gaz d'électrons. Nous allons essayer dans le paragraphe suivant d'en préciser l'origine physique.

\subsection{Les excitations collectives}

Mais revenons tout d'abord à la description simple d'un métal. Nous nous plaçons dans le cas (simplifié) d'une bande unique étroite décrite par le Hamiltonien suivant :

$$
H=\sum_{i, j, \sigma} t_{i, j} c_{i, \sigma}^{+} c_{j, \sigma}
$$

$t_{i, j}$ décrit l'amplitude du saut entre les sites $i$ et $j$, et donc en fait l'énergie cinétique. $n_{i, \sigma}=c_{i, \sigma}^{+} c_{i, \sigma}$ désigne l'opérateur nombre d'occupation dans l'état $(i, \sigma)$. A l'aide des opérateurs transformés de Fourier, on peut encore écrire $H$ sous la forme :

$$
H=\sum_{\mathbf{k}, \sigma} \epsilon_{\mathbf{k}} c_{\mathbf{k}, \sigma}^{+} c_{\mathbf{k}, \sigma} .
$$

L'état fondamental $|F\rangle$ est un "liquide de Fermi", défini par

$$
\begin{aligned}
& \left\langle n_{\mathbf{k}, \sigma}\right\rangle=1 \text { si } \epsilon_{\mathbf{k}, \sigma} \leq \epsilon_{F} \\
& \left\langle n_{\mathbf{k}, \sigma}\right\rangle=0 \text { si } \epsilon_{\mathbf{k}, \sigma} \geq \epsilon_{F}
\end{aligned}
$$

où $\epsilon_{F}$ est le niveau de Fermi, déterminé par le nombre d'électrons dans la bande de conduction. Dans ce modèle, les états du type :

$$
c_{\mathbf{k}+\mathbf{q}, \sigma}^{+} c_{\mathbf{k}, \sigma^{\prime}}|F\rangle
$$

décrivent des excitations magnétiques individuelles où un électron de spin $\sigma^{\prime}$ est transferré du niveau de Fermi vers un état de plus haute énergie et de spin $\sigma$. La susceptibilité s'écrit :

$$
\chi^{o}(\mathbf{q}, \omega)=\sum_{\mathbf{k}} \frac{\left\langle n_{\mathbf{k}+\mathbf{q}, \downarrow}\right\rangle-\left\langle n_{\mathbf{k}, \uparrow}\right\rangle}{\omega-\left(\epsilon_{\mathbf{k}+\mathbf{q}, \downarrow}-\epsilon_{\mathbf{k}, \uparrow}\right)} .
$$

L'analyse de cette expression (due à Lindhardt) montre que les excitations individuelles forment un continuum, qui dépend de la dispersion des électrons $\epsilon_{\mathbf{k}, \sigma}$ ainsi que du niveau de Fermi.

Les excitations collectives apparaissent dans le cadre de modèles plus complexes, comme par exemple le modèle de Hubbard, où l'on tient compte d'une interaction de Coulomb "minimale", notée $U$, qui tend à empêcher deux électrons de spin opposés de se trouver sur le même site (deux électrons de même spin ne peuvent pas se trouver au même endroit, du fait du principe de Pauli) :

$$
H=\sum_{i, j, \sigma} t_{i, j} c_{i, \sigma}^{+} c_{j, \sigma}+U \sum_{i} n_{i, \uparrow} n_{i, \downarrow}
$$



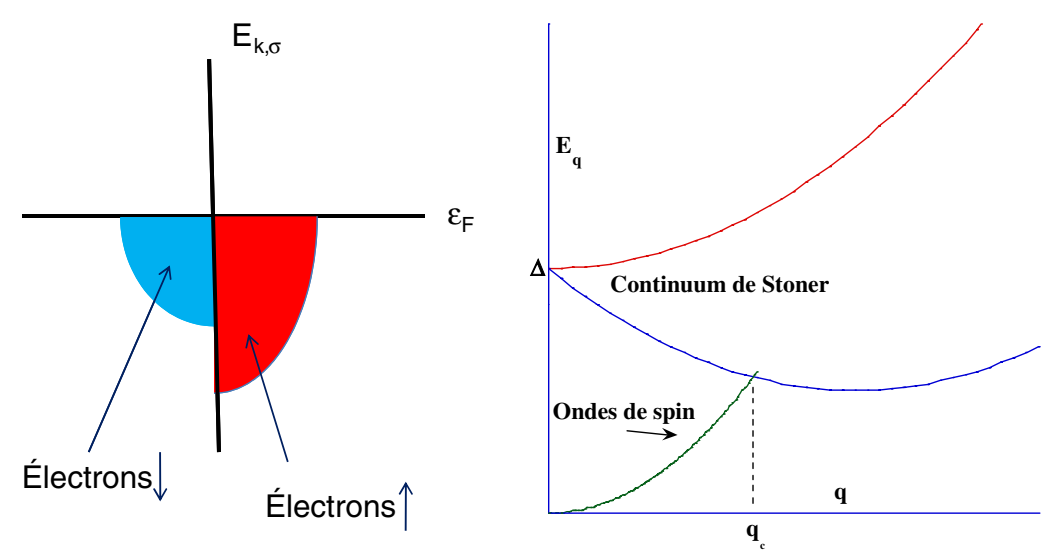

Figure 3. A gauche : schéma de bandes dans le modèle de Stoner. A droite : le continuum de Stoner et la branche d'excitations collectives.

Dans le cadre du modèle de Stoner, les corrélations peuvent stabiliser une phase ferromagnétique où les densités électroniques de spin $\uparrow$ et $\downarrow$ sont différentes. Cette différence est caractérisée par :

$$
\Delta=\left\langle n_{\uparrow}\right\rangle-\left\langle n_{\downarrow}\right\rangle \geq 0
$$

avec

$$
\left\langle n_{\sigma}\right\rangle=\frac{1}{N} \sum_{i}\left\langle n_{i, \sigma}\right\rangle
$$

L'énergie d'un électron dépend maintenant de son spin :

$$
E_{\mathbf{k}, \sigma}=\epsilon_{\mathbf{k}, \sigma}+U\left\langle n_{-\sigma}\right\rangle
$$

ce qui traduit l'apparition de 2 sous-bandes (voir la figure 3), mais les excitations individuelles correspondent toujours au transfert d'un électron de spin $\sigma$ situé au niveau de Fermi vers un état de plus haute énergie et de spin $\sigma^{\prime}$. Dans le cadre de la RPA, on cherche les excitations magnétiques sous la forme de combinaisons linéaires des excitations individuelles. Elles sont donc décrites par un opérateur:

$$
b_{\mathbf{q}}^{+}=\sum_{\mathbf{k}} \alpha_{\mathbf{k}} c_{\mathbf{k}+\mathbf{q}, \sigma}^{+} c_{\mathbf{k}, \sigma^{\prime}}=\sum_{\mathbf{k}} \frac{A}{\omega_{\mathbf{q}}-E_{\mathbf{k}+\mathbf{q}, \downarrow}+E_{\mathbf{k}, \uparrow}} c_{\mathbf{k}+\mathbf{q}, \downarrow}^{+} c_{\mathbf{k}, \uparrow}
$$

où les énergies $\omega_{\mathbf{q}}$ sont solutions de l'équation aux valeurs propres :

$$
1=U \sum_{\mathbf{k}} \frac{\left\langle n_{\mathbf{k}+\mathbf{q}, \downarrow}\right\rangle-\left\langle n_{\mathbf{k}, \uparrow}\right\rangle}{\omega_{\mathbf{q}}-\left(E_{\mathbf{k}+\mathbf{q}, \downarrow}-E_{\mathbf{k}, \uparrow}\right)}=U \chi_{o} .
$$

On montre qu'il existe des solutions telles que

$$
\omega_{\mathbf{q}} \approx E_{\mathbf{k}+\mathbf{q}, \downarrow}-E_{\mathbf{k}, \uparrow}
$$

et qui sont donc très proches des excitations individuelles. L'ensemble de ces excitations (quasi) individuelles forme un continuum, que l'on appelle continuum de Stoner. Il existe également une solution supplémentaire, qui apparait à petit q, et qui décrit une excitation "collective" dans le sens où les $\alpha_{\mathbf{k}}$ sont tous du même ordre de grandeur. Les calculs montrent que l'énergie $\omega_{\mathbf{q}}$ correspondante se comporte comme $\omega_{\mathbf{q}}=D q^{2}$ à petit $\mathbf{q}$. Elle est donc isotrope et quadratique en $q$. $D$ est la constante de raideur. En première approximation on montre que $D$ est proportionnel au nombre d'électrons dans 
la bande de conduction et à $U / \Delta$ c'est-à-dire à l'inverse de l'aimantation par atome. La susceptibilité magnétique a une forme typique de la RPA :

$$
\begin{aligned}
\chi & =\frac{\chi_{o}}{1-U \chi_{o}}, \\
\chi^{o}(\mathbf{q}, \omega) & =\sum_{\mathbf{k}} \frac{\left\langle n_{\mathbf{k}+\mathbf{q}, \downarrow}\right\rangle-\left\langle n_{\mathbf{k}, \uparrow}\right\rangle}{\omega-\left(E_{\mathbf{k}+\mathbf{q}, \downarrow}-E_{\mathbf{k}, \uparrow}\right)} .
\end{aligned}
$$

dont les pôles signalent la présence d'un mode collectif.

\section{MESURE DES NIVEAUX DE CHAMP CRISTALLIN DANS UNE TERRE RARE}

La diffusion inélastique des neutrons permet aussi d'étudier le magnétisme des terres rares et en particulier les niveaux de champ cristallin. Dans ce cas, le magnétisme est dû à l'opérateur moment cinétique total $\mathbf{J}$ et la section efficace des neutrons fait intervenir les composantes de l'opérateur $\mathbf{J}$ perpendiculaires à $\mathbf{Q}: \mathbf{J}_{\perp}$. Les $2 J+1$ niveaux correspondants sont définis par le couplage spin-orbite ainsi que par le champ cristallin. En fonction de la symétrie du champ cristallin, l'opérateur $\mathbf{J}$ va se transformer suivant le groupe d'espace attaché à cette symétrie. Les représentations irréductibles de ce groupe sont notées $\Gamma_{n}$ et correspondent aux fonctions d'onde $\left|\Gamma_{n}, v\right\rangle$ (l'indice $v$ distingue les fonctions sous-tendant les niveaux dégénérés). La section efficace de diffusion de neutrons s'écrit :

$$
\begin{aligned}
\frac{\partial^{2} \sigma}{\partial \Omega \partial E^{\prime}}= & \left(\gamma r_{o}\right)^{2} \frac{k^{\prime}}{k}\left|\frac{g}{2} F(\mathbf{Q}) e^{-W(\mathbf{Q})}\right|^{2} \\
& \times \sum_{n, n^{\prime}, v, v^{\prime}} p_{n}\left\langle\Gamma_{n}, \nu\left|\mathbf{J}_{\perp}\right| \Gamma_{n^{\prime}}, v^{\prime}\right\rangle\left\langle\Gamma_{n^{\prime}}, v^{\prime}\left|\mathbf{J}_{\perp}\right| \Gamma_{n}, v\right\rangle \delta\left(\omega-E_{n^{\prime}}+E_{n}\right)
\end{aligned}
$$

où $p_{n}=g_{n} e^{-\beta E_{n}}$ est la probabilité que le niveau $E_{n}$ soit peuplé ( $g_{n}$ est la dégénérescence du niveau). En fonction des éléments de matrice, on trouve des transitions plus ou moins fortes. Les fortes sont celles qui ont lieu près de $\mathbf{Q}=0$, c'est-à-dire celles permises par l'approximation dipolaire.

On remarque que cette expression ne dépend de $\mathbf{Q}$ que par l'intermédiaire du facteur de forme $F(\mathbf{Q})$ : en effet, si l'échange entre les ions est faible, ces transitions n'intéressent que des ions isolés.

Prenons l'exemple de l'ion $\operatorname{Pr} 3+$ en symétrie cubique. Cet ion se caractérise par l'état ${ }^{4} f_{2}, \ell=3$. Selon les règles de Hund pour une couche moins qu'à moitié remplie, $L=5, S=1, J=|L-S|=4$. Le champ cristallin en symétrie cubique éclate les 9 niveaux dégénérés en 4 niveaux. Le niveau $\Gamma_{1}$ est un état singulet, non magnétique; le niveau $\Gamma_{4}$ est un état triplet, le niveau $\Gamma_{3}$ un état doublet et le niveau $\Gamma_{5}$ un état triplet. L'application de la théorie des groupes permet aussi de calculer quelles sont les transitions permises ou interdites. Ceci est représenté sur la figure 4.

\section{QUELQUES MOTS SUR LES NEUTRONS POLARISÉS}

Il est souvent très utile de séparer la diffusion nucléaire (au sens de la diffusion par les noyaux des atomes) de la diffusion magnétique, sans parler de la diffusion magnétique par le spins des noyaux. Pour cela on dispose d'un outil irremplaçable : les faisceaux de neutrons polarisés. Depuis fort longtemps ils ont été utilisés en diffusion élastique pour la détermination des structures magnétiques complexes, pour dresser des cartes de densité de spin électronique dans des systèmes organiques complexes. Grâce aux développements et à l'amélioration de ces techniques, on peut aussi les utiliser en diffusion inélastique.

Dans une expérience de diffusion de neutrons polarisés, les neutrons sont préparés dans un état de spin donné $\uparrow$ ou $\downarrow$, à l'aide d'un monochromateur en alliage d'Heusler ou d'un guide supermirroir 
(a)
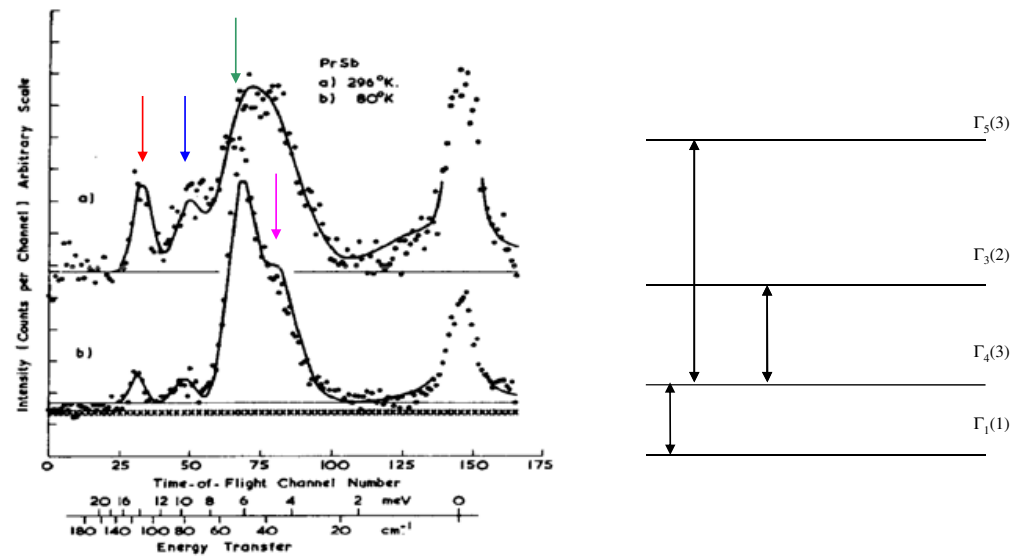

(b)

Figure 4. A gauche : Spectres neutroniques d'excitations de champ cristallin (ref. 7) Exemple de spectres permettant de déterminer les transitions entre niveaux de champ cristallin dans PrSb. Ces mesures sont faites sur un spectromètre à temps de vol, en gain d'énergie des neutrons. Elles sont extraites de : K.C. Tuberfield et al. J. Appl. Phys. 42 (1971) 1746 Neutron Crystal-Field Spectroscopy in Rare Earth Metallic Compounds. On identifie en énergie décroissante : $\Gamma_{5} \rightarrow \Gamma_{4}$ à $14.3 \mathrm{meV}, \Gamma_{5} \rightarrow \Gamma_{3}$ à $9.8 \mathrm{meV}, \Gamma_{4} \rightarrow \Gamma_{1}$ à $6.3 \mathrm{meV}$ et $\Gamma_{3} \rightarrow \Gamma_{4}$ pour $4.6 \mathrm{meV}$. A droite, représentation des transitions permises dans la symétrie cubique.

particulier appelé “bender”. On peut retourner ce spin à l'aide d'un dispositif appelé flipper. Après diffusion, on analyse non seulement l'énergie mais aussi la direction du spin des neutrons. On utilise pour cela un analyseur en alliage d'Heusler associé à un flipper placé sur le trajet des neutrons diffusés. On peut ainsi déterminer les sections efficaces des 4 processus $\uparrow \uparrow, \downarrow \downarrow, \uparrow \downarrow$ et $\downarrow \uparrow$ ( 2 non-spin-flip "NSF" et 2 spin-flip "SF").

Tout le long de leur trajet, on "guide" la polarisation des neutrons à l'aide d'aimants installés dans les "bras" du spectromètre : on parle de champ guide. Des bobines de Helmoltz placées autour de l'échantillon permettent de faire tourner "adiabatiquement" la polarisation incidente des neutrons pour la faire coincider au niveau de l'échantillon avec une direction particulière de l'espace. Bien entendu, la direction du vecteur $\mathbf{Q}$ est une direction remarquable dans une mesure de diffusion. Ce dispositif permet donc en particulier d'appliquer une polarisation parallèle ou perpendiculaire à $\mathbf{Q}$ (voir la figure 5).

\subsection{Approche simplifiée de l'analyse longitudinale (LPA)}

Les équations générales donnant la polarisation du faisceau de neutrons diffusé et la section efficace différentielle inélastique en fonction de la polarisation incidente sont décrites dans le cours de L.P Régnault donné dans une précédente école des JDNs. Nous ne donnons ici que les résultats principaux.

Les mesures sont effectuées en appliquant la polarisation incidente respectivement selon les 3 directions : $x$ parallèle à $\mathbf{Q}, y$, perpendiculaire à $\mathbf{Q}$ dans le plan de diffusion et $z$, perpendiculaire à $\mathbf{Q}$ hors du plan de diffusion. La technique d'analyse de polarisation longitudinale permet d'accéder expérimentalement à la projection de la polarisation finale $P_{f}$ sur la polarisation incidente. On définit les fonctions de corrélation suivantes :

$$
\begin{gathered}
N=\left\langle N_{\mathbf{Q}} N_{\mathbf{Q}}^{+}\right\rangle, \\
M_{a a}=\left\langle M_{\mathbf{Q} a} M_{\mathbf{Q} a}^{+}\right\rangle,
\end{gathered}
$$



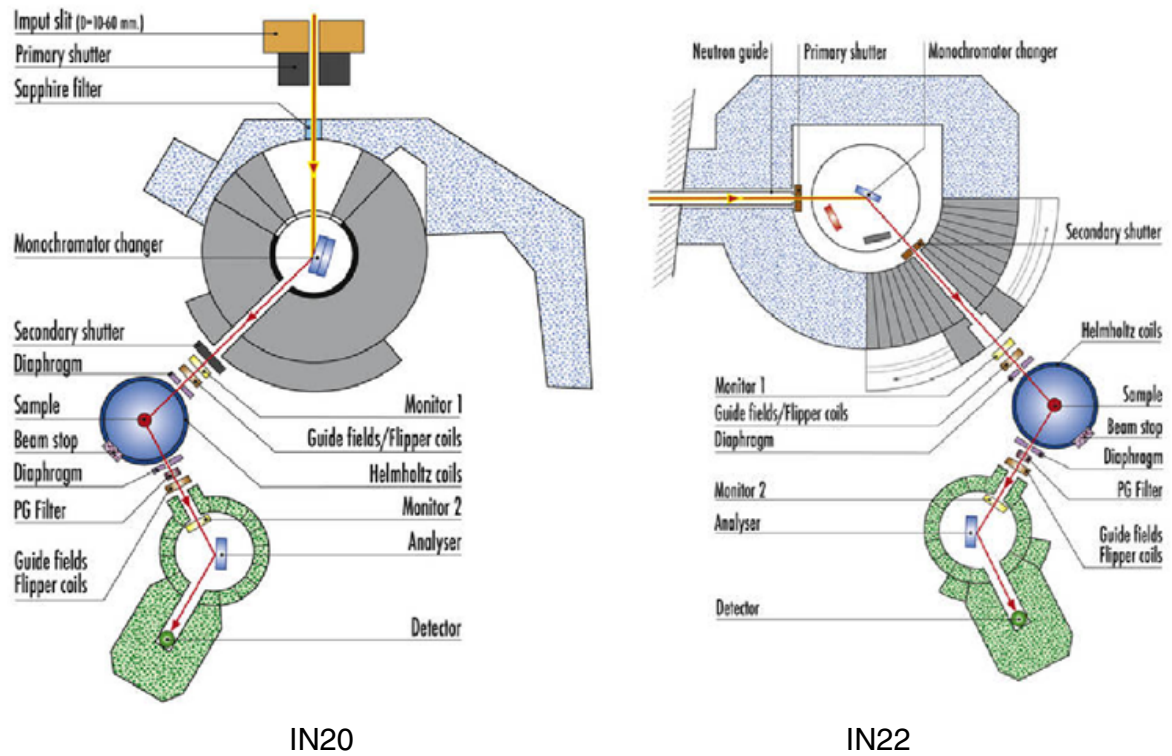

Figure 5. Schéma des spectromètres 3-axes à neutrons polarisés IN20 et IN22 (ILL-CRG).

$$
\begin{aligned}
M_{c h} & =i\left(\left\langle M_{\mathbf{Q} y} M_{\mathbf{Q} z}^{+}\right\rangle-\left\langle M_{\mathbf{Q} z} M_{\mathbf{Q} y}^{+}\right\rangle\right), \\
R_{a} & =\left\langle N_{\mathbf{Q}} M_{\mathbf{Q} a}^{+}\right\rangle+\left\langle N_{\mathbf{Q}}^{+} M_{\mathbf{Q} a}\right\rangle
\end{aligned}
$$

décrivant respectivement les fonctions de corrélation associées à la diffusion nucléaire, aux composantes de spin selon la direction $a$ (toujours perpendiculaires à $Q$ comme il se doit), au terme dit "chiral" $M_{c h}$, et enfin au terme d'interférence magnétique-nucléaire $R_{a}$. Il faut alors retenir que, selon la polarisation incidente parallèle à $x, y$ ou $z$, les sections efficaces des 4 processus s'écrivent :

$$
\begin{aligned}
\sigma_{x}^{\uparrow \uparrow} & \propto N, \\
\sigma_{x}^{\downarrow \downarrow} & \propto N, \\
\sigma_{x}^{\uparrow \downarrow} & \propto M_{y y}+M_{z z}-M_{c h}, \\
\sigma_{x}^{\downarrow \uparrow} & \propto M_{y y}+M_{z z}+M_{c h}, \\
\sigma_{y}^{\uparrow \uparrow} & \propto N+M_{y y}+R_{y}, \\
\sigma_{y}^{\downarrow \downarrow} & \propto N+M_{y y}-R_{y}, \\
\sigma_{y}^{\uparrow \downarrow} & \propto M_{z z}, \\
\sigma_{y}^{\downarrow \uparrow} & \propto M_{z z}, \\
\sigma_{z}^{\uparrow \uparrow} & \propto N+M_{z z}+R_{z}, \\
\sigma_{z}^{\downarrow \downarrow} & \propto N+M_{z z}-R_{z}, \\
\sigma_{z}^{\uparrow \downarrow} & \propto M_{y y}, \\
\sigma_{z}^{\downarrow \uparrow} & \propto M_{y y} .
\end{aligned}
$$


Par rapport à la diffusion inélastique de neutrons non polarisés, on voit donc toute la richesse de cette technique qui apporte des informations nouvelles. On remarque en outre que :

- La diffusion purement nucléaire (cohérente et incohérente due au désordre isotopique sans spin nucléaire), est toujours non spin-flip NSF. L'exemple qui est souvent donné est celui du nickel pour lequel aucun isotope ne possède de spin nucléaire.

- Si le noyau ne possède pas de spin, la différence entre les deux termes NSF d'une part et SF d'autre part, permet d'acceder à des quantités intéressantes, en particulier à $M_{y}, M_{z}$ et $M_{c h}$, ce qui peut être très utile pour résoudre les structures magnétiques.
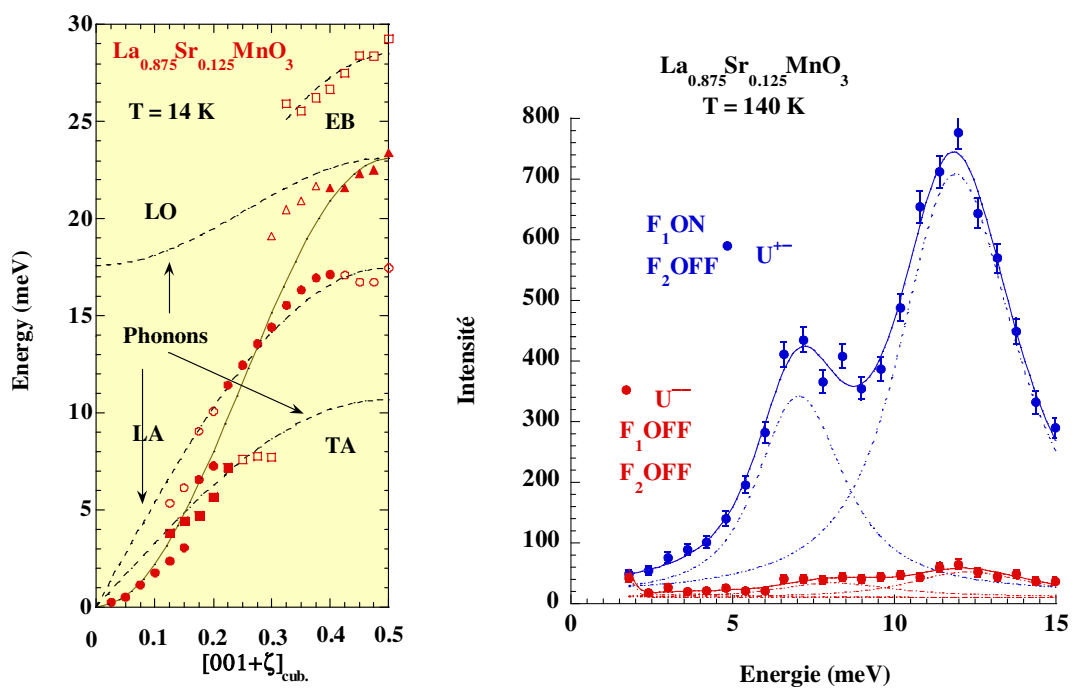

Figure 6. A gauche : courbes de dispersion des ondes de spin. A droite : spectres mesurés sur IN22 en $Q=$ $(0,0,1.25)$

En diffusion inélastique, les neutrons polarisés nous permettent le plus souvent de séparer ce qui est dû aux magnons ou plus généralement aux excitations magnétiques et ce qui est dû aux phonons. Dans l'étude des manganites perovskites, nous étions confrontés à des spectres complexes caractérisés par plusieurs branches magnétiques qui avaient des valeurs d'énergie très voisines de celles des phonons acoustiques et optiques. Les neutrons polarisés ont (parfois) permis de trancher. La figure 6 donne un exemple de scans mesurés sur IN22 au point $Q=(0,0,1.25)$ et à $k_{f}=2.662^{-1}$. Le monochromateur et l'analyseur sont tous deux des cristaux d'Heusler. Les flippers peuvent faire changer l'état de polarisation aussi bien avant qu'après l'échantillon. Les valeurs des énergies des magnons étant très proches de celles des phonons, on pouvait suspecter la présence d'un couplage magnon - phonon. Dans ce cas l'excitation devient mixte, et possède, à la fois, une composante magnétique et une composante nucléaire. Le spectre, présenté sur la figure 6 montre que la composante "nucléaire", déduide du canal $\sigma^{\downarrow \downarrow}$ (en rouge) est très faible, de l'ordre de la fuite de polarisation. Les ondes de spin sont observées dans le canal $\sigma^{\downarrow \uparrow}$ (en bleu). Nous en avons conclu qu'il n'y avait pas de couplage magnon - phonon classique.

\subsection{Le mode semi-polarisé}

Certaines expériences de neutrons polarisés peuvent aussi être menées en mode semi-polarisé. Il s'agit typiquement de l'étude de composés ferromagnétiques dont l'aimantation est saturée le long de l'axe x (parallèle à $\mathbf{Q}$ ) par un champ magnétique horizontal. Le monochromateur n'est pas polarisant (d'où 


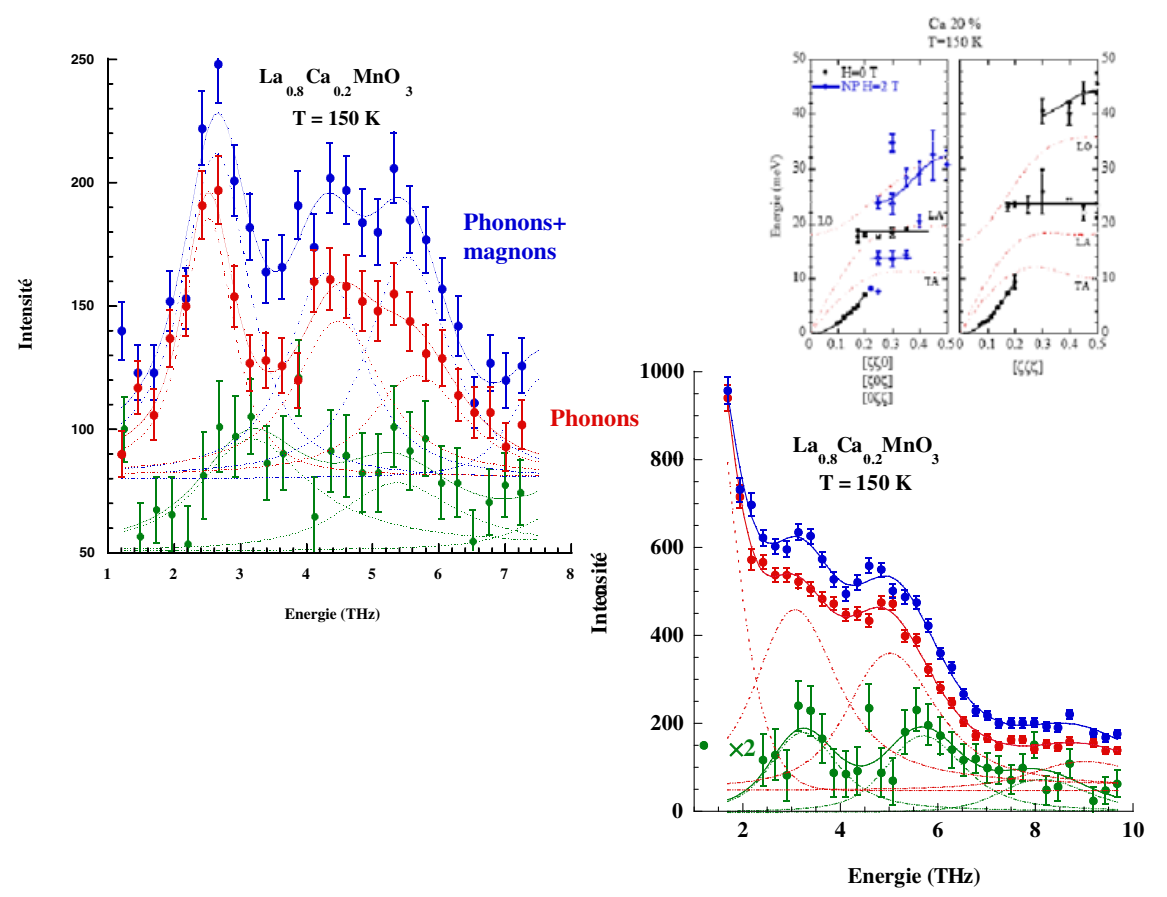

Figure 7. Exemple de mesures en mode semi-polarisé sur IN20 (ILL).

l'appellation semi-polarisé), mais en revanche, on utilise un analyseur en Heusler associé à un flipper. Dans le canal $\uparrow$, on mesure :

$$
\sigma_{x}^{\uparrow \uparrow}+\sigma_{x}^{\downarrow \uparrow}
$$

et dans le canal $\downarrow$ :

$$
\sigma_{x}^{\uparrow \downarrow}+\sigma_{x}^{\downarrow \downarrow}
$$

Parmi les processus SF, les seuls possibles dans cette configuration correspondent à $\sigma_{x}^{\downarrow \uparrow}$, où l'on créé une onde de spin, et où par conséquent l'aimantation est réduite. Les autres $\left(\sigma_{x}^{\uparrow \downarrow}\right)$ sont impossibles car on ne peut pas annihiler une onde de spin, ce qui reviendrait à augmenter l'aimantation (qui est déjà saturée). Si la statistique est suffisante, la différence entre les deux canaux permet donc d'isoler la seule contribution magnétique $\sigma_{x}^{\downarrow \uparrow}$.

La figure 7 présente des exemples de scans mesurés sur IN20 dans ce type de configuration. Le monochromateur (Si111) est non polarisant. L'analyseur est un alliage d'Heusler 111; grâce au flipper entre l'échantillon et l'analyseur, on peut compter les neutrons diffusés les canaux $\uparrow$ et $\downarrow$. La figure montre deux spectres mesurés à $\mathbf{Q}=(0.3,0.3,2)$ et $k_{f}=3^{-1}$. Dans le canal $\uparrow$ on ne mesure que les phonons (points rouges), dans le canal $\downarrow$ on mesure la totalité (points bleus). La différence des deux spectres permet d'acceder à la contribution des magnons (points verts sur la figure 7). On peut aussi étudier le spectre à plus haute énergie, en un point équivalent du réseau réciproque : $\mathbf{Q}=(1.3,1.3,0)$ et $k f=4.1^{-1}$. La résolution est moins bonne mais les intensités sont plus fortes et on atteint des valeurs d'énergie plus élevées. Il semblerait donc que le mode aux environs de $18 \mathrm{meV}$ appartienne plutôt à la branche de phonon longitudinale acoustique, mais la mesure manque de statistique pour une conclusion définitive. On mesure bien deux voire trois niveaux d'excitations magnétiques vers $3,5.5$ et $8 \mathrm{THz}$ soit 12, 23 et $33 \mathrm{meV}$. Le signal différence a été multiplié par 2 par rapport au spectre précédent. 


\section{CONCLUSION}

En conclusion, il faut retenir de ce cours que la section efficace de diffusion inélastique magnétique des neutrons s'écrit :

$$
\begin{aligned}
\frac{\partial^{2} \sigma}{\partial \Omega \partial E^{\prime}}= & \frac{k^{\prime}}{k}\left(\gamma r_{o}\right)^{2} \frac{1}{2 \pi \hbar} \sum_{\ell, \ell^{\prime}, d, d^{\prime}} e^{i \mathbf{Q}\left(\mathbf{R}_{\ell, d}^{o}-\mathbf{R}_{\ell^{\prime}, d^{\prime}}^{o}\right)} F_{d}(\mathbf{Q}) e^{-W_{\ell, d}} F_{d}^{*}(\mathbf{Q}) e^{-W_{\ell^{\prime}, d^{\prime}}} \\
& \times \int d t\left\langle\mathbf{S}_{\perp, \ell, d}(t=0) \cdot \mathbf{S}_{\perp, \ell^{\prime}, d^{\prime}}(t)\right\rangle e^{-i \omega t}
\end{aligned}
$$

Nous avons vu dans des cas simples, que les mesures permettent de déterminer la dispersion des excitations magnétiques collectives dans les solides. L'analyse de ces excitations permet de trouver les échelles d'énergie typique et donc de proposer une description quasi microscopique du système, en identifiant par exemple les différents termes d'un Hamiltonien. C'est là toute la force et la richesse des mesures de diffusion inélastique des neutrons.

\section{Références}

[1] Theory of neutron scattering from condensed matter (2 volumes) S.W. Lovesey (Oxford Science Publications) 1987.

[2] Methods of Experimental Physics vol.23 Part A-B-C Neutron Scattering Celotta-Levine 1986, Contributions de David L. Price, Kurt Sköld, Jean Rossat-Mignod, William G. Stirling, H. Glätlli et M. Goldman.

[3] Solid State Physics Aschroft/Mermin (Saunders College Publishing) 1976.

[4] J. Hubbard, Proc. Roy. Soc., 1963, p.A281-401.

[5] A. Blandin, Theory of Condensed Matter, IAEA, Vienna, 1968, p.691.

[6] B. Hennion and F. Moussa, Ann. Phys. 1972, t.7, p. 233-246.

[7] K.C. Tuberfield et al., Journal of Applied Physics 42 (1971) 1746.

[8] Ecole Thématique de la SFN : Neutrons Polarisés. N. Kernavanois, E. Ressouche, H. Schober, J.L. Soubeyroux Eds. EDP Sciences 2005.

[9] Cyrille Boullier, Thèse de l'Université Joseph Fourier de Grenoble 2005.

[10] F. Moussa and M. Hennion, in Colossal Magnetoresistive Manganites, edited by T. Chatterji (Kluwer, Dordrecht, 2004.

[11] M. Hennion and F. Moussa New Journal of Physics, 7, 84 (2005). 